\title{
Avaliação de escoamento pluvial em módulos de telhados verdes com diferentes substratos
}

\author{
Brenda Mello Franco ${ }^{a}$, Cacio Miranda Andresa, Júlia Konrada, Rutineia Tassia*, Tiago \\ Liberalesso $^{\mathrm{b}}$ \\ a Universidade Federal de Santa Maria, Rio Grande do Sul, Santa Maria, 97105-900, Brasil. rutineia@gmail.com \\ b Instituto Superior Técnico- Universidade de Lisboa, 1049-001, Lisboa, Portugal. \\ Recebido: 6 abril 2019 / Aceito: 4 maio 2019 / Publicado online: 27 maio 2019
}

\begin{abstract}
Resumo
A substituição de coberturas impermeáveis por coberturas verdes têm apresentado-se como uma alternativa ambientalmente adequada para a redução do escoamento superficial das águas da chuva. Nesse sentido, essa pesquisa avaliou a eficiência da composição do substrato de telhados verdes na redução do escoamento pluvial. Para tanto, foi realizado o monitoramento de eventos de chuva e vazão, considerando 40 sistemas experimentais (10 tratamentos, com 4 repetições em cada) de telhados verdes modulares, compostos por solo puro, substrato comercial puro e pela combinação de diferentes substratos: casca de arroz carbonizada, casca de arroz in natura e solo. Não houve diferença estatisticamente significativa na taxa de retenção média entre os tratamentos avaliados. Apesar disso, verificou-se que todos os tratamentos testados apresentaram taxa média de retenção do escoamento pluvial igual a 74\%, o que demonstra o potencial de utilização de resíduos como a casca de arroz na composição de substratos para telhados verdes, visto seu custo ser mais baixo que o custo de substratos comerciais, devido às suas propriedades como leveza, porosidade e boa drenagem, além de constituir-se em uma alternativa ambientalmente adequada para a destinação desse passivo ambiental.
\end{abstract}

Palavras-chave: Casca de arroz, Sedum rupestre, taxa de retenção.

\section{Evaluation of rainfall in modules of green roofs with different substrates}

\begin{abstract}
The replacement of impermeable coverings by green cover has been presented as an environmentally adequate alternative for the reduction of surface runoff from rainwater. In this sense this research evaluated the efficiency of the substrate composition of green roofs in the reduction of rainfall. Rainfall and flow events were monitored, considering 40 experimental systems (10 treatments, with 4 replications each) of modular green roofs, composed of pure soil, pure commercial substrate and by the combination of different substrates: carbonized rice husks, in natura rice husks and soil. There was no statistically significant difference in the mean retention rate among the evaluated treatments. Nevertheless, it was verified that all treatments tested presented a mean retention rate of the rainfall of $74 \%$, which demonstrates the potential of using residues such as rice husks in the composition of substrates for green roofs, since its cost is lower than the cost of commercial substrates, due to its properties such as lightness, porosity and good drainage, besides being an environmentally adequate alternative for the destination of this environmental liability.
\end{abstract}

Keywords: Rice husk, retention rate; Sedum rupestre.

\section{Introdução}

O ciclo hidrológico tem sofrido alterações devido ao desenvolvimento urbano desordenado, o que causa o aumento do escoamento superficial das águas da chuva, especialmente em razão do aumento da impermeabilização do solo. Esse fenômeno é proporcionado pelo emprego de superfícies impermeáveis, que atuam na redução das taxas de infiltração, o que por sua vez leva à diminuição das taxas de recarga para os aquíferos e à diminuição do escoamento básico (Klinkenborg, 2009).
Neste sentido, a substituição de coberturas impermeáveis por coberturas verdes têm apresentado-se como uma alternativa ambientalmente adequada. Estas coberturas verdes, também chamadas de telhados verdes, podem ser definidas como sistemas construtivos que possuem uma cobertura devidamente impermeabilizada, um sistema de drenagem para escoamento pluvial excedente, uma camada de solo e outra de vegetação.

Segundo Peck, S., Callaghan, C., Kuhn, M., Bass, B. (2002), na Europa, o telhado verde já é bastante empregado, 
tanto pela estética, como para a redução do consumo energético e para ajudar a reduzir o volume de escoamento pluvial. No Brasil, o uso de telhados verdes ainda é pouco difundido, mas traria muitos benefícios, principalmente nas regiões mais quentes, devido ao conforto térmico oferecido.

No que diz respeito ao escoamento pluvial, os telhados verdes absorvem e retêm a água da chuva, que fica interceptada e armazenada no substrato e no próprio sistema de drenagem. Assim, a capacidade de retenção do escoamento pluvial em telhados verdes é afetada, entre outros fatores, pela característica do substrato, sendo possível reduzir o escoamento pluvial entre $15 \%$ e $70 \%$ (Silva, 2011; Tassi, R., Tassinari, L. C., Piccilli, D. G. A., Persch, C. G., 2014).

Além disso, os substratos utilizados em coberturas verdes podem ser compostos por uma vasta gama de materiais alternativos, sejam eles elementos naturais ou sintéticos, que objetivam um equilíbrio entre as propriedades físicas ideais desejáveis, como baixa densidade, boa drenagem, e elevada capacidade de retenção de água, promovendo a reutilização destes resíduos (Ampim, P. A., Sloan, J. J., Cabrera, R. I., Harp, D. A., Jabers, F. H., 2010; Baldessar, 2012).

Diante disso, essa pesquisa buscou avaliar a eficiência da composição do substrato de telhados verdes na redução do escoamento pluvial.

\section{Material e Métodos}

\section{Características da Região Experimental}

Os módulos estão localizados na área experimental do grupo de pesquisa Ecotecnologias, situada nas coordenadas latitude $29^{\circ} 42^{\prime} 48^{\prime}$ ' S e longitude $53^{\circ} 43^{\prime} 07^{\prime}$ ' $\mathrm{O}$, dentro da área da Universidade Federal de Santa Maria (UFSM), no município de Santa Maria, região central do estado do Rio Grande do Sul. A região está inserida na unidade morfológica denominada Depressão Central, numa zona de transição entre os biomas Pampa e Mata Atlântica (Löbler, C. A., Sccoti, A. A., Werlang, M. K., 2015).

De acordo com a classificação de Köppen (1989), o clima de Santa Maria situa-se na área de clima temperado, chuvoso e quente. O clima é considerado do tipo mesotérmico brando, assim como a maior parte do estado, visto que as temperaturas não são tão baixas no inverno em relação a outras regiões de altitudes mais elevadas, a exemplo da Serra Gaúcha.

\section{Módulos experimentais}

Foi realizado o monitoramento quantitativo por meio da confecção de módulos experimentais que reproduziam telhados verdes extensivos. O conjunto de módulos foi composto por dez diferentes tratamentos em esquema de quatro repetições, totalizando 40 módulos. Estes módulos representam condições semelhantes a um telhado verde real, e permitem que seja avaliada a capacidade de retenção do escoamento pluvial durante cada evento.

Realizou-se o monitoramento do escoamento pluvial através dos módulos, juntamente com os dados de precipitação, no período de setembro de 2018 a março de 2019.

Os módulos experimentais foram montados em bombonas de plástico de $20 \mathrm{dm}^{3}$, as quais tiveram suas dimensões alteradas para $36 \mathrm{~cm}$ x $27 \mathrm{~cm}$ x $15 \mathrm{~cm}$, o que resulta em um valor 14,58 $\mathrm{dm}^{3}$ em cada bombona. Em cada módulo foram inseridos camada drenante, manta geotêxtil (filtro), meio de crescimento (substrato) e plantas.

A camada drenante inserida foi composta por uma geomembrana tridimensional, comercialmente conhecida como MacDrain 2L®, com $10 \mathrm{~mm}$ de filamentos de polipropileno e termosoldado, com espessura média da camada de $2 \mathrm{~cm}$ (Silva, 2010). Ainda, o material possui mais de $90 \%$ de espaços vazios elevando a capacidade drenante. Entre a geomembrana drenante e o meio de crescimento, foi colocada uma segunda manta geotêxtil com o intuito de reduzir o processo de colmatação e evitar a migração de partículas finas a partir do substrato para a camada de drenagem.

Na parte inferior de cada módulo, entre a geomembrana tridimensional e a manta geotêxtil, foi inserido um cano de PVC longitudinal com $25 \mathrm{~mm}$ de diâmetro, com a finalidade de conduzir a água excedente. $\mathrm{O}$ cano inserido possui perfurações sendo envolto por uma manta geotêxtil e, na sua extremidade, existe uma curva de $90^{\circ}$ de mesmo diâmetro na qual foi conectada a uma mangueira transparente $3 / 4$, que direciona a água aos recipientes coletores para posterior quantificação do volume percolado.

Em cada módulo experimental (Figura 1) foi colocado um volume de $9,72 \mathrm{dm}^{3}$ de substrato, com uma espessura de 10 $\mathrm{cm}$, dentro da faixa que corresponde os telhados verdes extensivos $(5-15 \mathrm{~cm})$. Os galões coletores, possuem volume de 5 litros, foram projetados para receber um volume de chuva de até $50 \mathrm{~mm}$, até a saturação total da camada de substrato.

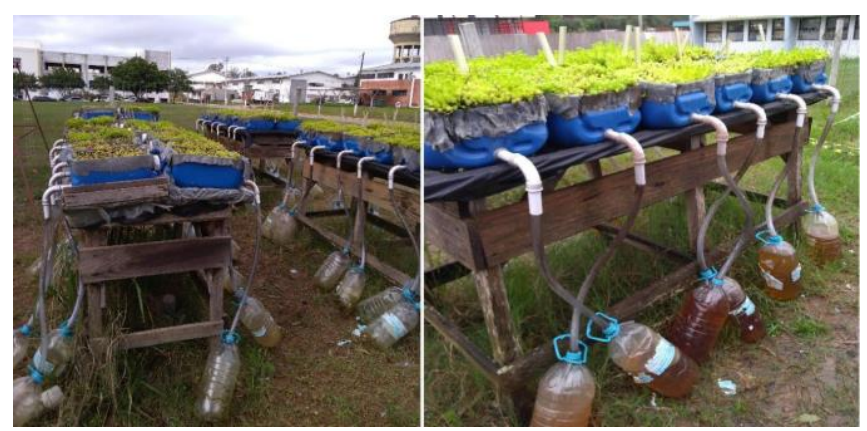

Figura 1. Módulos experimentais simulando condições de um telhado verde extensivo e sistema de captação do escoamento pluvial.

As plantas utilizadas foram da espécie Sedum rupestre L. subsp. elegans (Lej.) Hegi et. Plantas do gênero Sedum já são empregadas mundialmente em telhados verdes extensivos, por possuírem alta tolerância ao estresse hídrico e, consequentemente, sobreviver a condições restritivas, além de apresentarem adaptabilidade às condições climáticas locais (Jobim, 2013; Lorenzini Neto, 2014; Tassi et al., 2014).

Organismos da espécie Sedum rupestre L. subsp. elegans (Lej.) Hegi et. (Figura 2) estão aptos para armazenar muita 
água nas folhas, são resistentes ao estresse hídrico e se recuperam facilmente dos períodos de seca. A água fica retida no sistema solo-planta por alguns dias e alimenta as plantas por evaporação (Jobim, 2013).

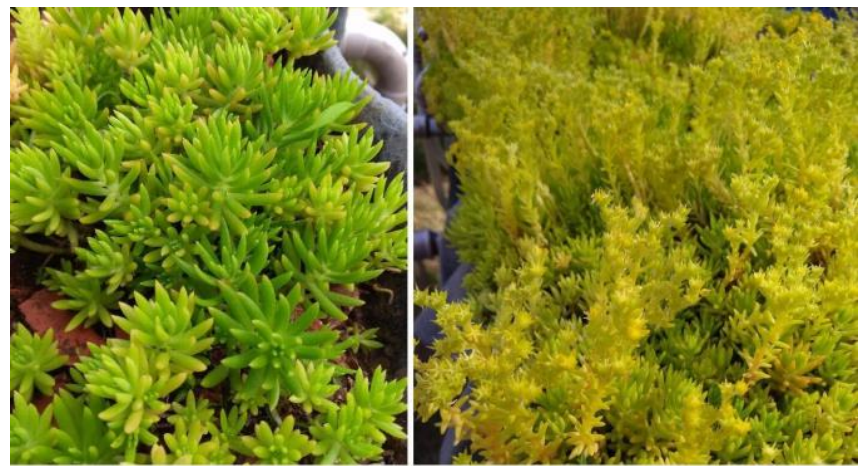

Figura 2. Sedum rupestre nas fases vegetativa (esquerda) e reprodutiva (direita).

As plantas utilizadas são oriundas de uma residência próxima ao local de instalação do experimento. O plantio foi realizado no mês de maio de 2017, ano que começou o desenvolvimento do experimento, com um espaçamento de $5 \mathrm{~cm}$ entre plantas, totalizando trinta mudas por módulo. Nas primeiras duas semanas pós plantio, período de aclimatação das mudas, os módulos foram mantidos ao abrigo da incidência direta de sol e chuva, sendo irrigados diariamente até o completo estabelecimento das mudas, para diminuir o estresse pós-transplante. Vinte dias após o transplante da vegetação, os módulos experimentais foram colocados sob condições naturais e dispostos em bancadas elevadas a $1 \mathrm{~m}$ do solo com declividade de $1 \%$.

\section{Delineamento da Pesquisa e Composição do Substrato}

$\mathrm{O}$ experimento em campo foi desenvolvido com delineamento experimental inteiramente casualizado (DIC), possuindo dez tratamentos e quatro repetições. Como controle, foi utilizado o substrato composto por $100 \%$ solo do Horizonte A, da classe Argissolo Vermelho-Amarelo e foi extraído de uma profundidade entre 10 a $40 \mathrm{~cm}$, coletado de um local próximo de onde foi instalado o experimento em campo. A camada superficial foi removida para evitar a importação de sementes ou restos vegetais capazes de regeneração, conforme orienta a FLL (2008). Também para fins comparativos, um dos tratamentos foi composto exclusivamente por substrato comercial Garden Plus®.

Os demais tratamentos foram constituídos por diferentes composições, previamente selecionados, sendo eles: casca de arroz carbonizada (CAC); casca de arroz in natura (CAN); vermicomposto (VMC); resíduo da construção civil (RCC); vermiculita; e solo, que foram combinados em diferentes proporções para compor os meios de crescimento. Todos os tratamentos foram testados e elaborados em laboratório (Tabela 1). As proporções de cada componente do substrato foram estimadas em volume (FLL, 2008). O volume do vermicomposto e vermiculita mantiveram-se fixos e as proporções de resíduo da construção civil foram fixadas em 20\%, como recomendado por Mickovski et al.
(2013).

Tabela 1. Percentual de cada tipo de substrato na composição dos tratamentos.

\begin{tabular}{cccccc}
\multirow{4}{*}{ Tipo } & & \multicolumn{4}{c}{ Composição (\%) } \\
\cline { 3 - 6 } & SC1 & 50 & 0 & 50 & 0 \\
\multirow{4}{*}{ CAC } & SC2 & 40 & 10 & 50 & 0 \\
& SC3 & 30 & 20 & 50 & 0 \\
& SC4 & 20 & 30 & 50 & 0 \\
& SC5 & 10 & 40 & 50 & 0 \\
\hline \multirow{4}{*}{ CAN } & SN3 & 30 & 20 & 50 & 0 \\
& SN4 & 20 & 30 & 50 & 0 \\
& SN5 & 10 & 40 & 50 & 0 \\
\hline \multirow{2}{*}{ REF } & SOLO & 0 & 100 & 0 & 0 \\
& SCOM & 0 & 0 & 0 & 100 \\
\hline
\end{tabular}

CA: casca de arroz; CAC: Casca de Arroz Carbonizada; CAN: Casca de Arroz Natural; Outros: Vermiculita (15\%), resíduo da construção civil (20\%) e vermicomposto (15\%); SCOM: Substrato comercial a base de turfa; SOLO: Argissolo Vermelho-Amarelo (Horizonte A); Os tratamentos SC possuem substrato formulado a partir de casca de arroz carbonizada; Os tratamentos $\mathrm{SN}$ possuem substrato formulado a partir de casca de arroz natural; REF: substratos usados como referência.

Optou-se pelo uso de resíduos da construção civil e da rizicultura, devido a disponibilidade local e a necessidade de uma destinação para estes materiais. Noya, M. G., Cuquel, F. L., Schafer, G., Armindo, R. A. (2017), Carrijo, O. A., Liz, R. S., Makishima, N. (2002) e Mickovski et al. (2013) já realizaram estudos que apontaram para o potencial de utilização destes resíduos.

O resíduo da construção civil utilizado no experimento foi obtido em obras de construções e reformas realizadas dentro do campus universitário da UFSM. O material selecionado foi triturado e peneirado, resultando em fragmentos com diâmetro entre $1 \mathrm{~mm}$ e $19 \mathrm{~mm}$. Quanto à casca de arroz utilizada no experimento, esta proveio de uma indústria de beneficiamento do grão localizada no bairro Camobi, distante, aproximadamente, $2 \mathrm{Km}$ da UFSM. Parte desse material foi carbonizado, enquanto que o restante foi utilizado sem nenhum tipo de tratamento prévio (in natura). $\mathrm{O}$ vermicomposto utilizado foi cedido pelo grupo de Biologia do Solo e do Ambiente, produzido no Setor de Minhocultura do Departamento de Solos da UFSM, a partir de esterco bovino.

\section{Capacidade de retenção hídrica}

A capacidade de retenção hídrica nos módulos experimentais foi quantificada através do monitoramento hidrológico em cada uma das unidades de telhado verde. Portanto, foram verificados os volumes de precipitação diária incidente e os volumes escoados em cada módulo após o evento de chuva. Ao todo, foram utilizados dados de 16 eventos de precipitação.

O volume de chuva foi mensurado a partir dos dados da estação meteorológica do Instituto Nacional de Meteorologia 
(INMET) instalada na área da UFSM. O volume de escoamento produzido por cada módulo experimental é drenado e armazenado nos galões coletores. Após cada evento de chuva, esse volume armazenado foi medido com auxílio de uma proveta graduada com capacidade máxima de $10 \mathrm{~mm}$.

A taxa de retenção é calculada utilizando-se a média do volume escoado das quatro repetições, por meio da Equação: $T R=P-V e P * 100$. Onde: TR a taxa de retenção para cada substrato $(\%), \mathrm{P}$ a precipitação total $(\mathrm{mm})$ e Ve o volume de escoamento total médio das quatro repetições $(\mathrm{mm})$.

Com os resultados obtidos dos cálculos da taxa de retenção de cada um dos 40 módulos para cada evento coletado, calculou-se a taxa de retenção média de cada tipo de tratamento.

Por fim, realizou-se a análise estatística do experimento por meio da ANOVA (Teste F), seguida do Teste de Tukey, para analisar a variância entre os tratamentos, utilizando o software R Studio (R Core Team, 2017).

\section{Resultados e Discussão}

As taxas de retenção nos diferentes tipos de composição de substratos (tratamentos) estão descritos na Tabela 2. Para a maioria das unidades experimentais, observou-se baixo coeficiente de variação, com valores mais alto para os tratamentos SC5 $(8,89 \%)$ e SC1 $(6,45 \%)$. Essa variabilidade pode ter ocorrido devido à variações na forma de incidência da chuva e do vento nos módulos, bem como à compactação do substrato, estrutura radicular e formação de caminhos preferenciais. Entretanto, as taxas médias de retenção (TRM) dos tratamentos não apresentaram variabilidade significativamente diferentes entre si, apresentando TRM igual a $74 \pm 4,8 \%$, indicando uma boa eficiência na retenção do escoamento pluvial.

Pesquisas anteriores utilizando substratos alternativos e coberturas vegetais semelhantes apresentaram resultados de TRM próximos aos encontrados nesta pesquisa. Tonial et al. (2017), que utilizou casca de arroz para composição do substrato e gramíneas do tipo esmeralda, obteve TRM igual a $62 \%$ das águas pluviais.

Savi e Tavares (2018) também fizeram uso da casca de arroz como substrato e as espécies Bulbine frutescens, Tradescantia zebrina, Arachis Repens e Sedum mexicanum como cobertura, as quais foram utilizadas por serem do tipo suculentas. Estes autores analisaram treze eventos de chuva não necessariamente sequenciais e obtiveram resultados importantes com relação a outros fatores, sendo a espécie Tradescantia zebrina a que apresentou valores mais semelhantes com a Sedum rupestre, com uma TRM da água pluvial igual a $73 \%$.

Tabela 2. Taxas de retenção de água de chuva em diferentes tipos de composição de substratos (tratamentos) utilizados em unidade experimental de telhados verdes

\begin{tabular}{cccccc}
\hline \multirow{2}{*}{ Tratamento } & \multicolumn{4}{c}{ Replicata* } & \multirow{2}{*}{ TMR } \\
\cline { 2 - 5 } & 1 & 2 & 3 & 4 & \\
\hline SC1 & $63,31 \pm 22,24$ & $77,52 \pm 19,75$ & $69,11 \pm 23,93$ & $75,47 \pm 22,92$ & $71,35 \pm 6,45$ \\
SC2 & $81,51 \pm 21,18$ & $79,53 \pm 18,40$ & $69,28 \pm 21,21$ & $70,85 \pm 31,59$ & $77,36 \pm 5,48$ \\
SC3 & $70,85 \pm 28,70$ & $64,84 \pm 25,62$ & $70,55 \pm 24,07$ & $70,79 \pm 23,11$ & $69,26 \pm 2,95$ \\
SC4 & $73,48 \pm 24,35$ & $77,12 \pm 25,10$ & $80,65 \pm 20,22$ & $73,09 \pm 24,45$ & $76,08 \pm 3,54$ \\
SC5 & $71,72 \pm 24,75$ & $71,50 \pm 23,48$ & $90,22 \pm 12,66$ & $74,96 \pm 20,89$ & $77,10 \pm 8,89$ \\
SN3 & $68,57 \pm 24,06$ & $75,66 \pm 22,50$ & $80,87 \pm 16,55$ & $72,17 \pm 22,03$ & $74,32 \pm 5,24$ \\
SN4 & $79,07 \pm 17,56$ & $78,00 \pm 18,99$ & $72,41 \pm 21,89$ & $76,02 \pm 27,38$ & $76,38 \pm 2,93$ \\
SN5 & $73,54 \pm 19,63$ & $69,78 \pm 22,99$ & $71,72 \pm 27,12$ & $73,55 \pm 22,54$ & $72,14 \pm 1,80$ \\
SOLO & $74,57 \pm 26,48$ & $75,40 \pm 23,72$ & $76,24 \pm 23,98$ & $72,89 \pm 21,30$ & $75,03 \pm 1,01$ \\
SCOM & $72,89 \pm 19,66$ & $69,65 \pm 24,94$ & $76,73 \pm 19,25$ & $74,43 \pm 23,56$ & $73,42 \pm 2,97$ \\
\hline
\end{tabular}

Composição dos tratamentos: SC1: 50:50 (CA:CM), onde, CA -Casca de Arroz e CM: 15\% Vermiculita, 20\% resíduo da construção civil e 15\% vermicomposto; SC2: 40:10:50 (CA:Solo:CM), onde o solo foi o Argissolo Vermelho-Amarelo (Horizonte A); SC3: 30:20:50 (CA:Solo:CM); SC4: 20:30:50 (CA:Solo:CM); SC5: 10:40:50 (CA:Solo:CM); SN3: 10:40:50 (CN:Solo:CM), onde CN é a Casca de Arroz Natural; SN4: 20:30:50 (CN:Solo:CM); SN5: 10:40:50 (CN:Solo:CM); SCOM: Substrato comercial a base de turfa. TMR - Taxa média de retenção (\%) de cada tratamento. *cada média da taxa de retenção (\%) das replicatas foi calculada a partir de 16 eventos de chuva.

Santos e Castilho (2018), reportaram valores de TRM igual a $73 \%$ com o uso de herbáceas floríferas para cobertura vegetal e fibra de coco, casca de arroz carbonizado, turfa e vermiculita expandida na composição dos substratos.

Na pesquisa deOliveira (2012), foram obtiddas TRM iguais a $77 \%$ e $81 \%$, para os substratos $\mathrm{S} 1(15 \%$ solo $+55 \%$ coco $+30 \%$ componentes comerciais) e $\mathrm{S} 2(30 \%$ solo $+40 \%$ coco $+30 \%$ componentes comerciais), sendo Arachis pintoi a espécie utilizada como cobertura vegetal.

Por fim, o experimento realizado por Jobim (2013), reportou valor de TRM igual a $88,1 \%$ utilizando substrato de argila expandida paragrama São Carlos, como cobertura vegetal.

Os resultados aqui encontrados para a casca de arroz reportou que a sua aplicação para a finalidade aqui apresentada tem eficiência equivalente àqueles substratos tradicionais, podendo representar mais um fim útil para a casca de arroz, que é um subproduto produzido em volumes bem expressivo no beneficiamento do arroz.

A utilização de materiais alternativos possibilita o 
aprimoramento da técnica de aplicação de telhados verdes de modo a torná-la mais popular e sustentável do ponto de vista econômico e ambiental.

\section{Conclusões}

Não houve diferença significativa entre os tratamentos analisados, com taxa média de retenção geral em cerca de $75 \%$. Assim, observa-se o potencial de utilização da casca de arroz na composição de substratos para telhados verdes, visto seu custo ser mais baixo que o custo de substratos comerciais e também devido às suas propriedades como leveza, porosidade e boa drenagem. $\mathrm{O}$ uso da casca de arroz como material agregado em substratos para telhados verdes também constitui-se em uma alternativa ambientalmente adequada para a destinação desse passivo ambiental.

\section{Referências}

Ampim, P.A.Y.; Sloan, J. J., Cabrera, R. I., Harp, D. A. H., \& Jabers, F. H. (2010). Green Roof Growing Substrates: Types, Ingredients, Composition And Properties. Journal Of Environmental Horticulture, 28(4), 244-252. Recuperado de https://hrijournal.org/doi/abs/10.24266/0738-2898-28.4.244.

Baldessar, S.M.N. (2012). Telhado verde e sua contribuição na redução da vazão da água pluvial escoada (Dissertação de mestrado). Universidade Federal do Paraná, Curitiba, Paraná. Recuperado de https://acervodigital.ufpr.br/bitstream/handle/1884/52621/R\%20$\% 20 \mathrm{D} \% 20-$

\%20SILVIA\%20MARIA\%20NOGUEIRA\%20BALDESSAR.pdf?seq uence $=1 \&$ is Allowed $=y$.

Carrijo, O.A, Liz, R.S., \& Makishima, N. (2002). Fibra da casca do coco verde como substrato agrícola. Horticultura Brasileira, 20(4), 533-535. doi: http://dx.doi.org/10.1590/S0102-05362002000400003.

FLL - Forschungsgesellschaft Landschaftsentwicklung Landschaftsbau. (2008). Guidelines for the Planning, Construction and Maintenance of Green Roofing, English ed. Recuperado de http://www.greenrooftechnology.com/_literature_98993/Introduction_t o_the_German_FLL-

Guideline_for_the_Planning,_Execution,_and_Maintenance_of_GreenRoof_/.

Jobim, L.A. (2013). Diferentes tipos de telhados verdes no controle quantitativo da água pluvial, Santa Maria (Dissertação de mestrado). Universidade Federal de Santa Maria, Santa Maria, Rio Grande do Sul. Recuperado https://repositorio.ufsm.br/bitstream/handle/1/7842/JOBIM,\%20ALAN $\%$ 20LAMBERTI.pdf?sequence $=1$.

Klinkenborg, V. (2009). Green Roofs. Revista National Geographic. Recuperado de http://ngm.nationalgeographic.com/2009/05/greenroofs/klinkenborg-text/.

Löbler, C.A., Sccoti, A.A.V., \& Werlang, M.K. (2015). Contribuição à delimitação dos biomas Pampa e Mata Atlântica no município de Santa Maria, RS. Revista Eletrônica em Gestão, Educação e Tecnologia Ambiental, 19(2), 1250-1257. doi: https://doi.org/105902/2236117016038/.

Lorenzini Neto, F. (2014). Modelagem de telhado verde: uma análise da eficiência no controle do escoamento pluvial em diferentes escalas (Dissertação de Mestrado. Universidade Federal de Santa Maria, Santa Maria, Rio Grande do Sul. Recuperado de https://repositorio.ufsm.br/bitstream/handle/1/7860/LORENZINI\%20N ETO $\% 2 \mathrm{c} \%$ 20FRANCISCO.pdf?sequence $=1$ \&isAllowed $=\mathrm{y}$.

Mickovski, S.B., Buss, K., Mckenzie, B.M. \& Sökmener, B. (2013). Laboratory study on the potential use of recycled inert construction waste material in the substrate mix for extensive green roofs. Ecological Engineering, 61(Part $\quad \mathrm{C}), \quad 706-714 . \quad$ doi: http://dx.doi.org/10.1016/j.ecoleng.2013.02.015/.

Noya, M.G., Cuquel, F. L., Schafer, G., \& Armindo, R.A. (2017). Substrates for cultivating herbaceous perennial plants in extensive green roofs. Ecological Engineering, 102, 662-669. doi: https://doi.org/10.1016/j.ecoleng.2017.02.042/.

Oliveira, C.C. (2012). Substratos para uso em telhados verdes: Avaliação da retenção hídrica e qualidade da água de escoamento (Dissertação de
Mestrado). Universidade do Estado do Rio de Janeiro, Rio de Janeiro, Rio de Janeiro. Recuperado de http://www.bdtd.uerj.br/tde_busca/arquivo.php?codArquivo=8181.

Peck, S., Callaghan, C., Kuhn, M., \& Bass, B. (1999). Greenbacks from green roofs: forging a new industry in Canada. Canada Mortgage and Housing Corporation. Recuperado de http://techne17.pini.com.br/engenhariacivil/162/sistemas-construtivos-287779-1.aspx.

R Core Team. (2017). R: A language and environment for statistical computing. $R$ Foundation for Statistical Computing, Vienna, Austria. Recuperado de https://www.R-project.org/.

Santos, P.L.F., \& Castilho, R.M.M. (2018). Floriferous herbaceous and substrates for use on extensive green roofs. Ornamental Horticulture, 24(3), 261-268. doi: http://dx.doi.org/10.14295/oh.v24i3.1251.

Savi, A.C., \& Tavares, S.F. (2018). Telhados verdes: uma análise da influência das espécies vegetais na retenção de água de chuva. Revista de Arquitetura IMED, $\quad 7(1), \quad 50-67 . \quad$ Recuperado de https://seer.imed.edu.br/index.php/arqimed/article/view/2647/1869.

Silva, F.B. (2010). Telhado verde alveolar. Téchne Revista do Engenheiro Civil, Ed. 162. Porto Alegre, Rio Grande do Sul. Recuperado de https://commons.bcit.ca/greenroof/files/2012/01/Greenbacks.pdf.

Silva, T.F., Paiva, A., \& Santos, S. (2015). Capacidade de retenção de água em um telhado verde: Estudo de caso em Caruaru. XXI Simpósio Brasileiro de Recursos Hídricos. Recuperado de https://www.ufpe.br/documents/616030/926767/Capacidade_de_retenca o_em.pdf/3d627709-0857-4eef-b0f7-d761d946c889.

Tonial, M. et al (2017). Telhados verdes: uma perspectiva contemporânea. $\begin{array}{llll}\text { Revista CIATEC, } & 9(1), & 46-57 . & \end{array}$ https://doi.org/10.5335/ciatec.v9i1.6281.

Tassi, R., Tassinari, L.C.S., Piccilli, D.G.A., \& Persch, C.G. (2014). Telhado verde: uma alternativa sustentável para gestão das águas pluviais. Ambiente Construído, 14(1), 139-154. doi: http://dx.doi.org/10.1590/S1678-86212014000100012.

\section{Licença Creative Commons CC BY 4.0}

Este artigo foi publicado com acesso aberto para distribuíção sob os termos do Licença de Atribuição Creative Commons, que permite uso irrestrito, distribuição, e reprodução em qualquer meio, desde que o trabalho original seja devidamente citado. 